EPJ Web of Conferences 16, 06001 (2011)

DOI: $10.1051 /$ epjconf/20111606001

(C) Owned by the authors, published by EDP Sciences, 2011

\title{
Brown dwarfs in wide-field surveys
}

\author{
N. Lodieu ${ }^{1,2,3, a}$ \\ 1 Instituto de Astrofísica de Canarias, Vía Láctea s/n, 38200 La Laguna, Tenerife, Spain \\ 2 Departamento de Astrofísica, Universidad de La Laguna, 38205 La Laguna, Tenerife, Spain \\ 3 Ramón y Cajal fellow funded by the Spanish Ministry of Science and Innovation
}

\begin{abstract}
In this invited talk, I briefly summarise early photometric and proper motion surveys carried out in the nearest and youngest open clusters to introduce the motivation behind the Galactic Cluster component of the UKIRT Infrared Deep Sky Survey. Afterwards, I focus on the latest results that we obtained in the Upper Sco association and in the Pleiades. To finish, I show a comparison of the luminosity and mass functions obtained in the Upper Sco association, the Pleiades cluster, and $\sigma$ Orionis from the homogeneous set of data publicly available from the Galactic Clusters Survey.
\end{abstract}

\section{INTRODUCTION}

The knowledge of the stellar and substellar Initial Mass Function (IMF; [44, 55, 56]) is of prime importance to understand the formation of stars and brown dwarfs. Large samples of member candidates in several regions with a wide range of properties (age, density, environment, etc...) are required to accurately evaluate the shape and the slope of the IMF. Ultimately, those candidates should be confirmed photometrically, spectroscopically as well as though proper motion and radial velocity (and other means if possible) to confirm unambiguously their membership. With this main scientific objectives in mind, many large-scale optical photometric and proper motion surveys have been undertaken in various regions. The UKIRT Infrared Deep Sky Survey (UKIDSS) Galactic Clusters Survey (GCS) represents a new generation of large-scale surveys in the near-infrared, targeting thousands of square degrees in 10 regions to shed light on the universality of the IMF.

In Section 2, I give a brief summary of wide-field surveys conducted in young open clusters to put the UKIDSS GCS into context. In Section 3, I describe the UKIDSS project with a special emphasis on the GCS. In Sections 4 and 5, I summarise our results in the Upper Sco association and the Pleiades cluster, respectively. Finally, in Section 6, I compare the luminosity and mass functions derived in three regions imaged by the GCS: Upper Sco, the Pleiades, and $\sigma$ Orionis.

\section{WIDE-FIELD SURVEYS}

This section does not pretend to provide a full history of wide-field surveys targeting open clusters but a brief outline to introduce the main motivation behind the concept of the UKIDSS GCS. I would divide previous optical surveys in two main categories:

- Type I: availability of two epochs with a large baseline (>10 years) and two filters (e.g. $R$ and $I$ )

ae-mail: nlodieu@iac.es

This is an Open Access article distributed under the terms of the Creative Commons Attribution-Noncommercial License 3.0, which permits unrestricted use, distribution, and reproduction in any noncommercial medium, provided the original work is properly cited. 
- Type II: pure photometric surveys with two filters complemented by near-infrared data (at least one filter)

The first type makes use of photomgraphic plates (first and second Palomar Observatory Sky Survey POSS I \& POSS II) and is biased towards nearby young clusters with a proper motion several sigma above the mean motion of field stars present along the line of sight. Among the most popular clusters, I should cite the Pleiades [1, 19, 25, 27], Alpha Per [49], Hyades [53, 54], and Praesepe [2, 28, 32].

The second type of large-scale surveys also applies to the aforementioned clusters like the Pleiades [20, 46], Alpha Per [4], Hyades [9, 22], Praesepe [17, 24, 47] but also to regions with small proper motions i.e. hard to distinguish from field stars on a few year baseline. For example, we can mention $\sigma$ Orionis [8, 13], IC 4665 [12], NGC 2547 [26], IC2391 [4], Collinder 359 [35], to cite only a few.

More recently, several groups have made use of near-infrared photometry and, in particular, of the Two Micron All Sky Survey (2MASS; $[18,57])$ to select cluster member candidates. Again, two different but complementary methods have been used to infer the luminosity and mass functions over large areas.

- A proper motion study using photographic plates as first epoch and 2MASS as a second epoch combined with a photometric selection in the $(J-K, K)$ colour-magnitude diagram. This method was successfully applied to the Pleiades [1] and Praesepe [2].

- A pure statistical approach using control fields located a few degrees away from the cluster centre and observed in the same manner in order to estimate the level of contamination. This procedure was applied in $\sigma$ Orionis, IC 348, and the Pleiades [62].

These studies are very useful to probe the large scale structure of clusters, determine globally their mass functions, and study possible variations radially. Nevertheless, they suffer from two major drawbacks: firstly, photographic plates are not deep enough to reveal the full substellar population, secondly, nearinfrared filters alone are not ideal to distinguish young cluster members from old field stars because the sequence of $\mathrm{M}$ dwarfs in the $(J-K, K)$ diagram is vertical (see e.g. Fig. 2 in Adams et al. 2001).

\section{THE UKIDSS GALACTIC CLUSTERS SURVEY}

UKIDSS [34] $]^{1}$ made of five sub-surveys, is designed to reach three to four magnitudes deeper than 2MASS and cover thousands of square degrees in several infrared filters. The UKIDSS project is described in Lawrence et al. (2007; [34]) and uses the Wide Field Camera (WFCAM; [14]) installed on the UK InfraRed Telescope (UKIRT) and the Mauna Kea Observatory (MKO; [63]) photometric system described in Hewett et al. (2006; [31]). The pipeline processing is described in Irwin et al. (in prep) ${ }^{2}$ and the WFCAM Science Archive (WSA) in Hambly et al. (2008; [30]). Regular European-wide data releases are announced roughly every six months through WSA and those data become public to the entire world 18 months later.

The GCS, one of the UKIDSS components, aims at probing young brown dwarfs in ten star-forming regions and open clusters over $\sim 1000$ square degrees in five passbands $(Z Y J H K)$ across the $1.0-$ 2.5 micron wavelength range with a second epoch in $K$. The main goal is to constrain the slope of the IMF in the low-mass and substellar regimes to tackle important issues, including the formation and spatial distribution of low-mass stars and brown dwarfs. Early results on the IMF in the Pleiades and in Upper Scorpius are presented in Lodieu et al. (2007c; [38]) and Lodieu et al. (2007a; [37]), respectively.

\footnotetext{
1 www.ukidss.org

2 Extensive details on the data reduction of the UKIDSS images is available at http://casu.ast.cam.ac.uk/surveysprojects/wfcam/technical
} 


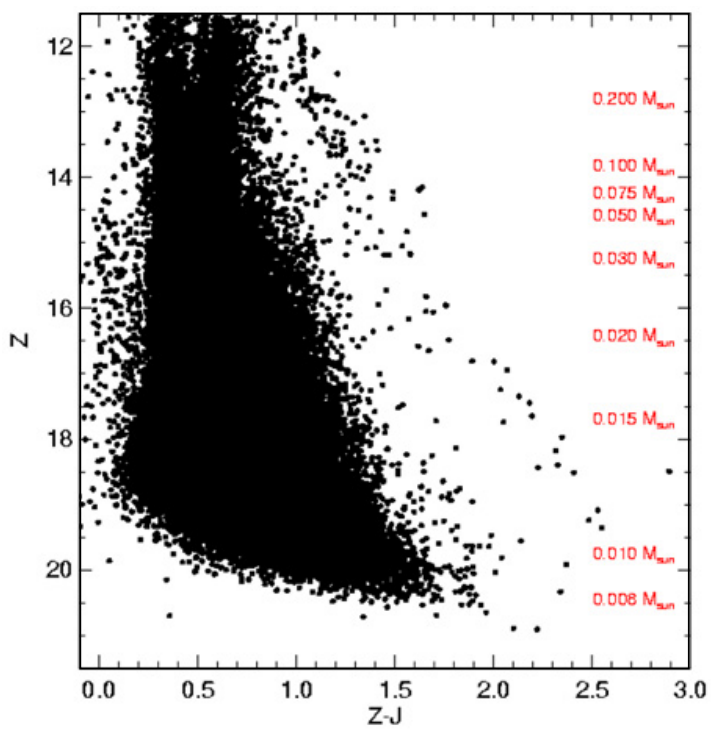

Figure 1. $(Z-J, Z)$ colour-magnitude diagram for 6.5 square degree covered in Upper Sco within the science verification phase of the UKIDSS GCS. The gap between the members of the association and field stars is clearly demarcated. The mass scale is shown on the right hand side of the diagrams and extends below $0.01 \mathrm{M}_{\odot}$, according to theoretical models. From Lodieu et al. (2007a).

\section{RESULTS IN THE UPPER SCO ASSOCIATION}

\subsection{Upper Sco}

Upper Sco is part of the Scorpius Centaurus association which is the nearest OB association to the Sun, located at a distance of $145 \pm 2$ pc derived from Hipparcos proper motion and parallax measurements [11]. The central part of the Upper Sco cluster is free of extinction $\left(A_{V} \leq 2\right.$ mag; [50]) and star formation has already ended [64] contrary to the Trapezium Cluster and Taurus. Multi-wavelength surveys have been extensively conducted in the region to uncover stellar and substellar members, including X-rays surveys with Einstein and ROSAT [51, 64], as well as optical and infrared surveys $[3,36,42,58]$. Preibisch \& Zinnecker (2002 [52]) derived the cluster IMF in the $2.0-0.1 \mathrm{M}_{\odot}$ mass range and found it consistent with the field IMF $[16,33]$. Several tens of brown dwarfs are currently known in Upper Sco [36, 42, 58, 59], the coolest have effective temperatures around $1800 \mathrm{~K}$ and masses around $0.008 \mathrm{M}_{\odot}[39]$.

\subsection{Upper Sco seen by the GCS}

The GCS is $100 \%$ complete down to $J=18.7 \mathrm{mag}$ and has surveyed $\sim 19$ square degrees in five passbands $(Z Y J H K)$ in Upper Sco. This survey is three magnitudes deeper than 2MASS and deeper than any previous optical survey in the region, reaching $0.008 \mathrm{M}_{\odot}$ according to state-of-the-art models [15] at $5 \mathrm{Myr}$ and $\mathrm{d}=145 \mathrm{pc}$. We found that the cluster sequence is well separated from field stars in the $(Z-J, Z)$ colour-magnitude diagram (Fig. 1) over the $0.3-0.008 \mathrm{M}_{\odot}$ mass range. From a limited area of 6.5 square degrees taken during the science verification phase of the GCS, we have selected a total of 129 photometric candidates below $0.3 \mathrm{M}_{\odot}$ based on their location in several colour-magnitude and two-colour diagrams $[36,37]$. We have confirmed the membership of 116 of them with proper motions derived from the cross-correlation between the GCS and 2MASS with an accuracy better than 10 mas/yr. The spatial distribution of candidates looks fairly homogeneous although there might be a hint of a 


\section{EPJ Web of Conferences}

concentration around the bright OB stars, fact also suggested by Slesnick et al. (2008). Additionally, we have derived the mass function in the $0.3-0.01 \mathrm{M}_{\odot}$ mass range (see Section 6), an order of magnitude deeper than the previous study [52].

The main improvements brought by the GCS with respect to previous surveys can be summarised as follows:

1. extraction of tenths of new brown dwarfs, yielding a significant increase to the current census of substellar objects in Upper Sco [36, 37]

2. discovery of 18 new brown dwarfs below $20 \mathrm{M}_{\text {Jup }}$, the limit of previous surveys in the region

3. photomery and proper motion for candidates with masses greater than $0.015 \mathrm{M}_{\odot}$ from the crossmatch between 2MASS (first epoch) and the GCS (second epoch)

4. extension of the mass function in Upper Sco from $0.1 \mathrm{M}_{\odot}$ down to $0.01 \mathrm{M}_{\odot}$ [37]

5. the discovery of the first L dwarfs confirmed by near-infrared cross-dispersed spectroscopy with the GNIRS spectrograph on Gemini (Fig. 2; [39]).

\section{RESULTS IN THE PLEIADES}

\subsection{The Pleiades}

The Pleiades cluster represents an ideal hunting ground for brown dwarfs and for the study of the mass function because: it is nearby ( $\mathrm{d}=130 \mathrm{pc}$; see e.g. [60]), young (age $=125 \mathrm{Myr}$; [61]), presents a large proper motion $(\sim 50 \mathrm{mas} / \mathrm{yr})$, and has low extinction $(\mathrm{E}(B-V)=0.03 \mathrm{mag})$. Several wide-field proper motion surveys have targeted the Pleiades in the past (e.g. [27]). Moreover, Adams et al. (2001; [1]) conducted a similar analysis with 2 MASS over the central $10 \times 10$ degree of the Pleiades. Their extensive multi-object spectroscopic follow-up suggested a contamination level below 13\%, much lower than the $30 \%$ estimated from pure photometric selections [45]. Deacon \& Hambly (2004; [19]) extended the work by Hambly et al. (1993; [27]) using the large baseline from the SuperCOSMOS Sky Survey to infer the mass function down to the hydrogen-burning limit. All these results point towards a log-normal mass function that flattens around $0.2 \mathrm{M}_{\odot}$.

\subsection{The Pleiades seen by the GCS}

We have cross-correlated 12 square degrees surveyed in $Z Y J H K$ in the Pleiades by the GCS with previous optical surveys from the Isaac Newton Telescope (INT; [20]) and Canada-France-Hawaii Telescope (CFHT; [46]) to extract proper motion members from 0.6 to $0.03 \mathrm{M}_{\odot}$ [38]. Our selection of cluster candidates has recovered a large number of published Pleiades members, in particular all the bright ones with masses greater than $0.1 \mathrm{M}_{\odot}[1,19,27]$. The outcome of our work is three-fold:

1. discovery of 70 new brown dwarfs with masses as low as $0.03 \mathrm{M}_{\odot}$ (Fig. 3), including $50 \%$ with proper motion membership [38]

2. determination of the brown dwarf binary fraction of $33-40 \%$ in the $0.075-0.030 \mathrm{M}_{\odot}$ mass range using the sample of high probability proper motion members as well as the photometric sample [38]. This estimate is in agreement with Monte-Carlo simulations of Maxted et al. (2005) and on the upper side of the estimate from the radial velocity survey conducted by Basri \& Reiners (2006). The inferred binary frequency is, however, on the low side of the photometric estimate by Pinfield et al. (2003; [48]) and significantly higher than predictions by high-resolution imaging surveys (e.g. [10]). The separations and mass ratios seem, however, consistent with findings for ultracool old field dwarfs and Pleiades brown dwarfs

3. determination of the Pleiades luminosity function using the sample of photometric candidates with membership probabilities. We have inferred a mass function in the $0.56-0.03 \mathrm{M}_{\odot}$ and fitted 
Research, Science and Technology of Brown Dwarfs and Exoplanets
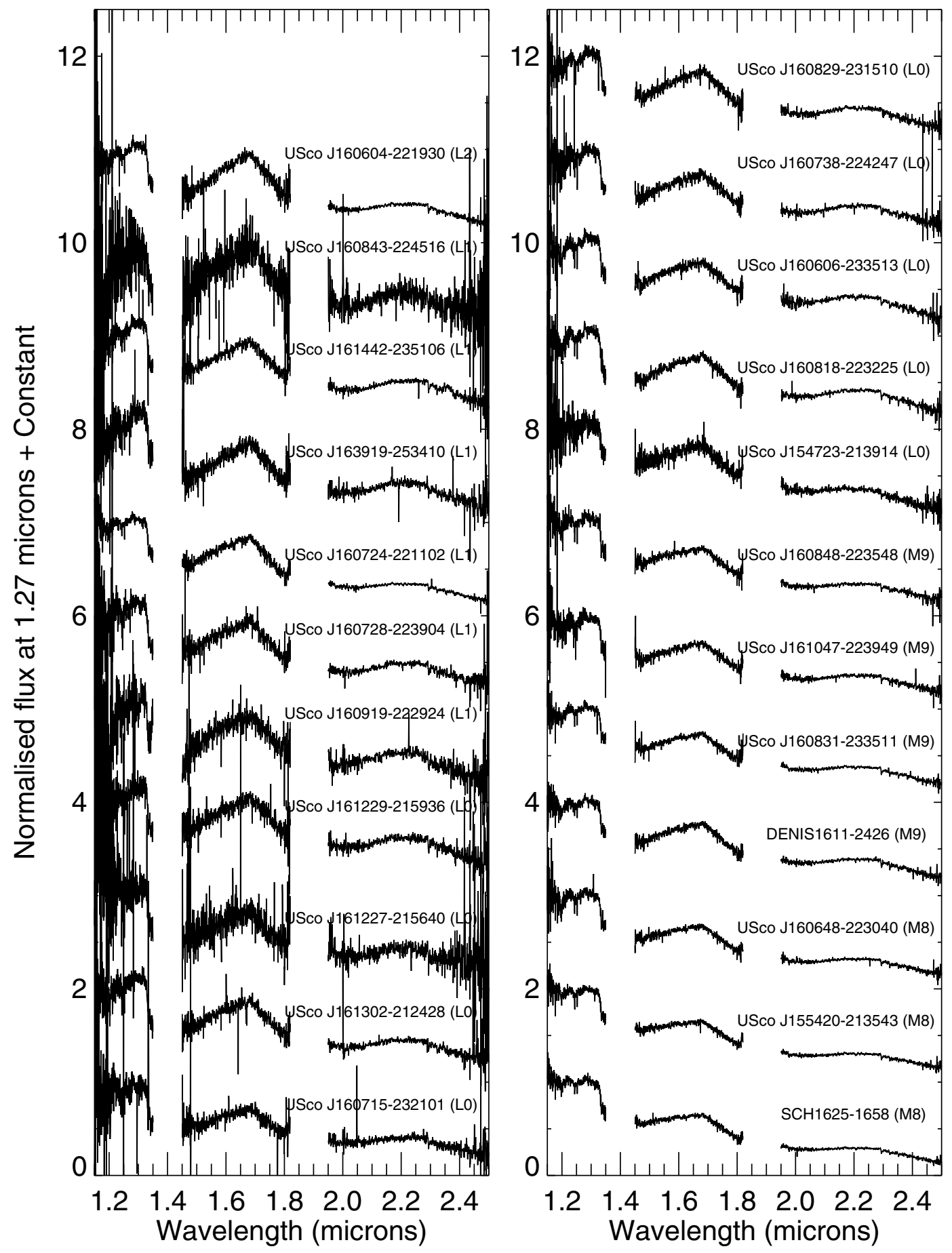

Figure 2. Cross-dispersed near-infrared $(1.15-2.5 \mu \mathrm{m})$ spectra of new Upper Sco brown dwarfs confirmed as spectroscopic members. Two known members are included for comparison: SCH162528-165850 (M8; Slesnick et al. 2008) and DENIS161103-242642 (M9; Martín et al. 2004). The names of the objects and their associated spectral types are marked above the spectra. New members are ordered by increasing spectral types. Spectra are normalised at 1.27 microns and shifted along the y-axis. From Lodieu et al. (2008). 


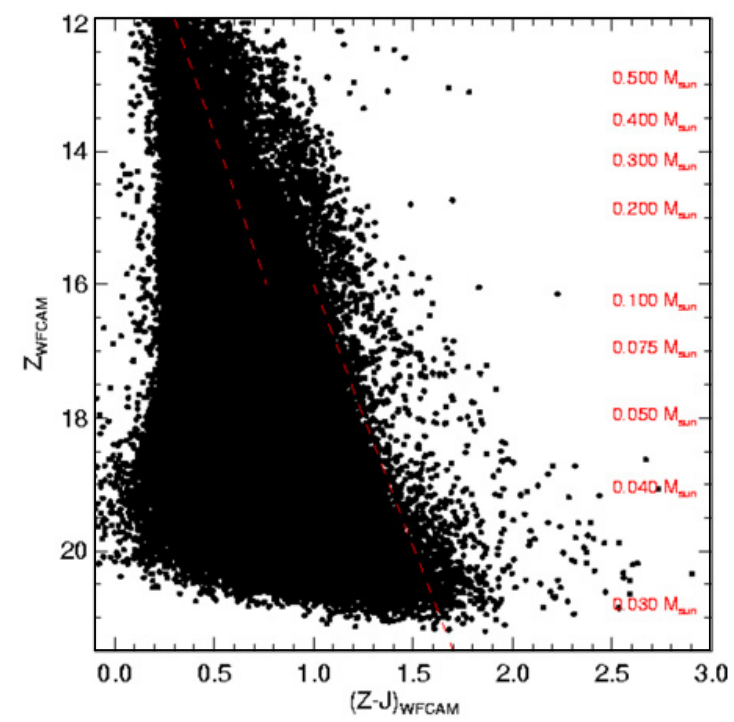

Figure 3. $(Z-J, Z)$ CMD for 12 square degrees in the Pleiades extracted from the UKIDSS GCS Data Release 1. The mass scale is shown on the right hand side and extends down to $0.03 \mathrm{M}_{\odot}$, according to theoretical models. The photometric selection criteria applied to select new candidates in the cluster prior to the derivation of proper motions is shown as a red dashed line. From Lodieu et al. (2007b).

a lognormal function peaking at $0.24 \mathrm{M}_{\odot}[38]$ which in agreement with previous studies in the cluster $[20,46]$.

\section{THE LUMINOSITY AND MASS FUNCTIONS}

In this section, I provide a comparison of the luminosity and mass functions in three clusters: the central 30 arcmin of $\sigma$ Orionis, 6.5 square degree in the Upper Sco association, and 12 square degrees in the Pleiades. Before moving into the interpretation of the plots displayed in Fig. 5, we summarise the selection of the member candidates in each cluster. Indeed, although the selection procedure is very similar and makes use of an homogeneous set of photometric data, I shall be point out some differences.

1. All selection procedures made use of 2MASS as first epoch to calculate proper motions accurate to $10 \mathrm{mas} / \mathrm{yr}$ down to $J=15.5 \mathrm{mag}$

2. All Upper Sco photometric candidates below $\sim 0.03 \mathrm{M}_{\odot}$ have been followed-up spectroscopically in the near-infrared: 21 out of 23 are confirmed as young late-M and early-L members of the association, making the faint end of the mass function fairly reliable

3. The selection of faint $(J \geq 15.5 \mathrm{mag}$ ) candidates in the Pleiades is not only photometric but also based on proper motions thanks to the $\sim 5$ year baseline between previous optical (INT+CFHT) surveys and the GCS. Therefore, the Pleiades mass function is derived from a full photometric and proper motion sample

4. The sequence of candidates in $\sigma$ Orionis is fairly well separated from the field stars, as in Upper Sco. However, the new faint candidates not reported earlier in the literature, lack proper motion and spectroscopy to confirm their membership. Hence, the mass function is purely photometric beyond $\sim 0.03 \mathrm{M}_{\odot}[40]$ 

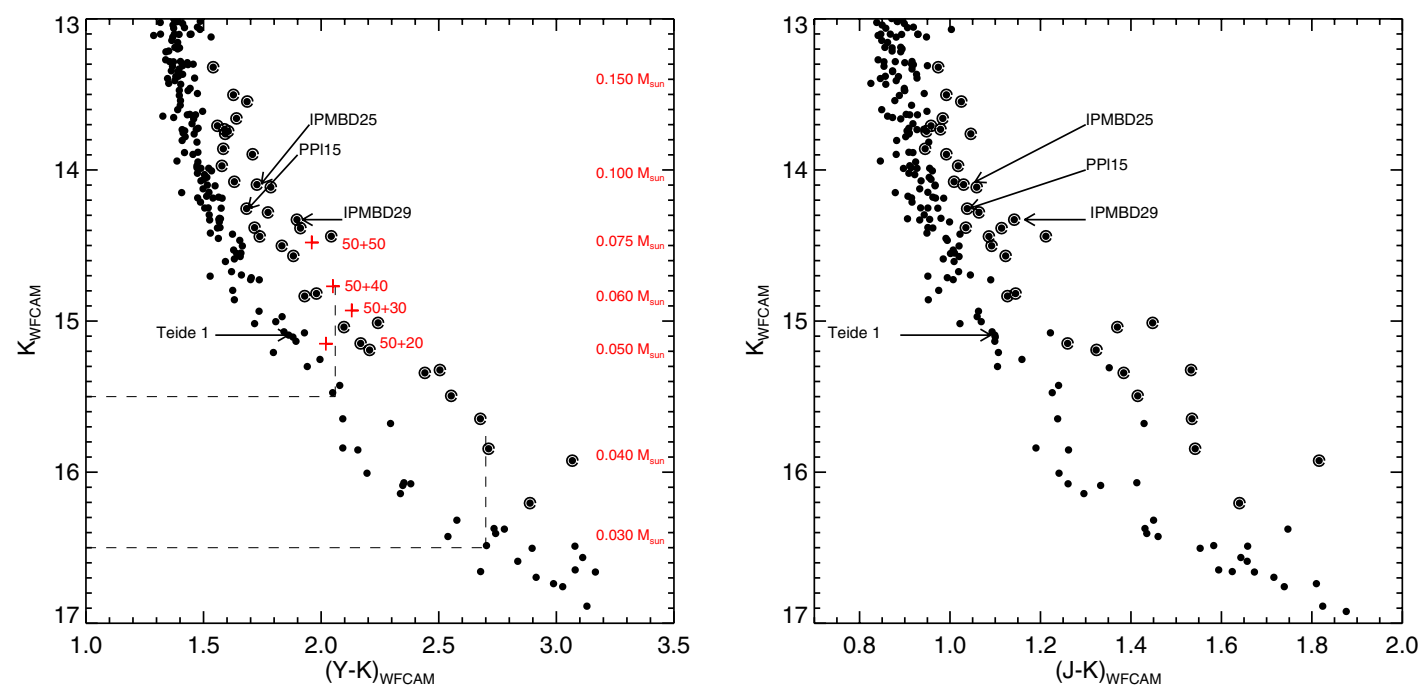

Figure 4. $(Y-K, K)$ and $(J-K, K)$ colour-magnitude diagrams for all substellar Pleiades candidates identified in the UKIDSS GCS. Substellar photometric multiple system candidates, originally picked out in the $(Y-K, K)$, are highlighted with an open circle around filled dots. We have confirmed all of them from their location in the $(J-K, K)$ diagram. We have found a total of 23 photometric multiple systems out of 63 brown dwarf candidates in the substellar regime $\left(K \geq 14.5 \mathrm{mag}\right.$ or $\left.\mathrm{M} \leq 0.075 \mathrm{M}_{\odot}\right)$, yielding a photometric binary fraction of 33-40\%. Known Pleiades binaries are highlighted, including PPl 15, a spectroscopic binary brown dwarf with a mass ratio of 0.85 [6], IPMBD 25, and IPMBD 29 (Bouy et al. 2006) as well as Teide 1 which sits in the single star sequence and for which no companion has been reported to date.

\subsection{The luminosity function}

The left hand side plot in Fig. 5 shows the luminosity function in $\sigma$ Orionis $(d=352 \mathrm{pc})$, Upper Sco $(\mathrm{d}=145 \mathrm{pc})$, and the Pleiades $(\mathrm{d}=130 \mathrm{pc})$ i.e. the number of objects as a function of the absolute $J$ magnitude. Error bars are Gehrels errors [23] rather than Poissonian error bars because the former represent a better approximation to the true error for small numbers. The upper limit is defined as $1+(\sqrt{(} d N+0.75))$ and the lower limit as $\sqrt{(} d N-0.25)$, assuming a confidence level of one sigma.

Three peaks are visible in the luminosity function of $\sigma$ Orionis at $\mathrm{M}_{J} \sim 5.25,7.25$, and $9.25 \mathrm{mag}$. The latter two peaks are also seen in the luminosity function presented in Caballero et al. (2007; [13]), shown as star symbols in Fig. 5. In Upper Sco and the Pleiades, we observe two peaks with a dip around $\mathrm{M}_{J}=9.5$ and $10.5 \mathrm{mag}$, respectively, likely due to the M7-M8 gap [21]. We should emphasize that the luminosity function of Upper Sco is "smoothed" as we are counting the number of objects per magnitude bin by steps of $0.5 \mathrm{mag}$ [37]. The difference in the number of peaks could arise from the different mass intervals probed due to the variety of ages: $125 \mathrm{Myr}$ for the Pleiades, $5 \mathrm{Myr}$ for Upper Sco, and $3 \mathrm{Myr}$ for $\sigma$ Orionis.

\subsection{The mass function}

We have transformed the observed magnitudes into masses using the NextGen [5] and DUSTy [15] models. The right hand side plot in Fig. 5 compares the mass function of $\sigma$ Orionis (filled symbols; $3 \mathrm{Myr}$; [40]) with the Upper Sco (open squares; $5 \mathrm{Myr} ; 145 \mathrm{pc}$; [37]) and the Pleiades (open triangles; $125 \mathrm{Myr} ; 130 \mathrm{pc}$; [38]) mass functions. Error bars are Gehrels error bars [23].

The Pleiades mass function is best fit by a lognormal function peaking at $0.24 \mathrm{M}_{\odot}$ in the logarithmic units originally used by Salpeter [55]. In linear units (see Fig. 5), the Pleiades mass function rises 

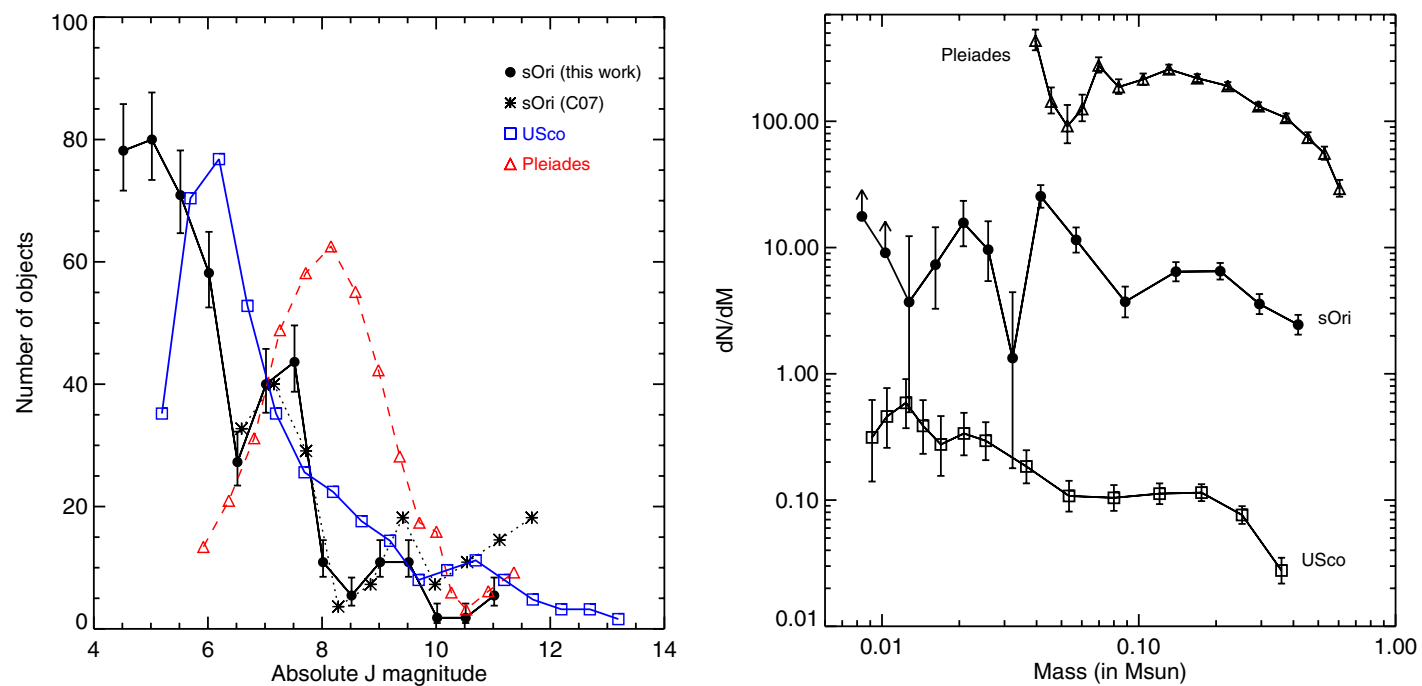

Figure 5. Left: The luminosity functions i.e. number of objects per absolute magnitude bin, assuming a distance of $352 \mathrm{pc}$ for $\sigma$ Orionis, $145 \mathrm{pc}$ for Upper Sco, and $130 \mathrm{pc}$ for the Pleiades. Right: The mass functions for the three clusters in linear units $\left(\mathrm{dN} / \mathrm{dM} \propto \mathrm{M}^{-\alpha}\right)$. The error bars and lower limits (arrows) are shown. The mass functions are shifted along the $y$-axis for clarity.

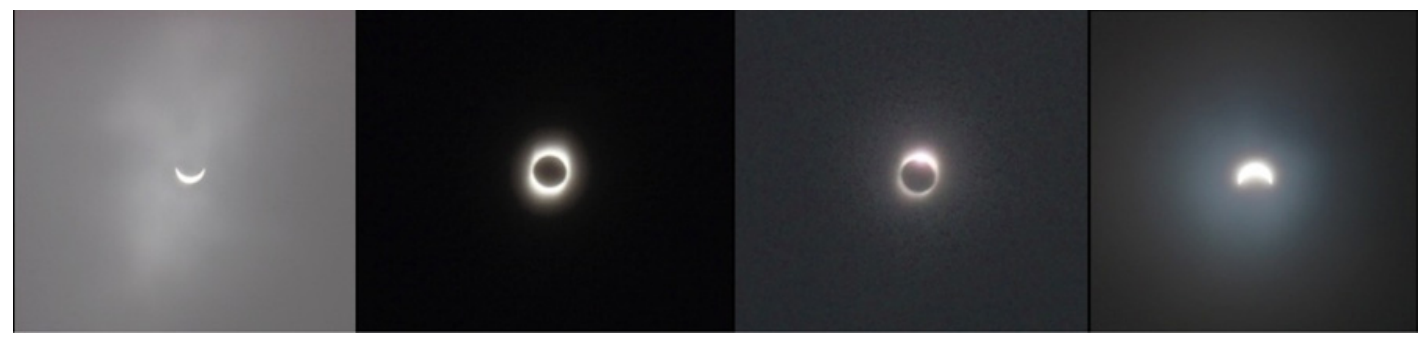

Figure 6. Sequence of the 5 min-long total solar eclipse witnessed on 2009 August $22^{\text {nd }}$ from the Moganshan mountain. Photos were taken with a digital camera Olympus Sylus 9000, equipped with an optical zoom of 10× but no tripod was used. Copyright: Nicolas Lodieu, Rohit Deshpande, Eduardo Martín, and Ramarao Tata.

towards lower masses and shows a strong dip around $0.05 \mathrm{M}_{\odot}$, attributed to the M7/M8 gap and the change in the properties of the atmospheres of brown dwarfs [21] and in particular the onset of dust condensation. The same conclusion can be drawn for the mass function in $\sigma$ Orionis but the dip occurs around $0.03 \mathrm{M}_{\odot}$. The index of the power law fit to the $\sigma$ Orionis and Upper Sco mass functions is comparable within the error bars over the same mass range, from low-mass stars down to the deuterium-burning limit. A dip is seen in the Upper Sco colour-magnitude diagrams around $Z=15.5 \mathrm{mag}\left(J \sim 14 \mathrm{mag} ; \mathrm{M} \sim 0.03 \mathrm{M}_{\odot}\right.$; Fig. 1$)$ but does not appear in the mass function due to the smoothing of the luminosity function (dictated by the small number statistics as we surveyed a small percentage of the full association; [37]). All these mass functions match the extrapolation of the field mass function in a log-normal form $[16,33]$.

\section{CONCLUSIONS}

I have presented a summary of our latest results in two regions imaged by the UKIDSS Galactic Clusters Survey: Upper Sco and the Pleiades. Combining the five GCS passbands and using 2MASS as first 
epoch to compute proper motion allowed us to identify low-mass stars, brown dwarfs, and planetarymass objects in Upper Sco and $\sigma$ Orionis. We have discovered the first L dwarfs in the Upper Sco association and have estimated photometrically the substellar binary fraction in the Pleiades. Finally, we have determined the luminosity and mass functions in Upper Sco, the Pleiades, and $\sigma$ Orionis.

The next step consists of applying the same procedure to the remaining regions targeted by the GCS, including Taurus, Orion, Praesepe, Hyades, IC 4665, Alpha Per, Per-OB2, and Coma Ber. The advent of VISTA (Visible and Infrared Survey Telescope for Astronomy), a near-infrared camera installed on a 4-m class telescope in Paranal (Chile) and offering a 1.6 square degree field-of-view on the sky, will build on the experience gained by the GCS and enlarge the variety of clusters. Furthermore, the combination of the large field-of-view and sensitivity offered by VISTA will allow us to initiate a widefield surveys in several regions to look for young $\mathrm{T}$ dwarfs and investigate the issue of the fragmentation limit in star formation [41].

I would like to thank Eduardo Martín for the original idea of organising a conference overlapping with the solar eclipse. I also would like to thank Eduardo, Rohit and Ramarao for the great trip to the Moganshan mountain where we enjoyed the full 5 minute-long solar eclipse. This work was partly funded by UK PPARC and by the Spanish Ministry of Science and Innovation through the Ramón y Cajal fellowship number 08-303-01-02.

This work is based in part on data obtained as part of the UKIRT Infrared Deep Sky Survey. The United Kingdom Infrared Telescope is operated by the Joint Astronomy Centre on behalf of the Science and Technology Facilities Council of the U.K.. Near-infrared spectra were obtained at the Gemini Observatory, which is operated by the Association of Universities for Research in Astronomy, Inc., under a cooperative agreement with the NSF on behalf of the Gemini partnership: the National Science Foundation (United States), the Particle Physics and Astronomy Research Council (United Kingdom), the National Research Council (Canada), CONICYT (Chile), the Australian Research Council (Australia), CNPq (Brazil) and CONICET (Argentina).

This research has made use of the Simbad database, operated at the Centre de Données Astronomiques de Strasbourg (CDS), and of NASA's Astrophysics Data System Bibliographic Services (ADS). This publication has also made use of data products from the Two Micron All Sky Survey, which is a joint project of the University of Massachusetts and the Infrared Processing and Analysis Center/California Institute of Technology, funded by the National Aeronautics and Space Administration and the National Science Foundation.

\section{References}

[1] Adams, et al. 2001, AJ, 121, 2053

[2] Adams et al. 2002, MNRAS, 333, 547

[3] Ardila et al. 2000, AJ, 120, 479

[4] Barrado y Navascués et al. 2002, A\&A, 395, 813

[5] Baraffe et al. 1998, A\&A, 337, 403

[6] Basri \& Martín 1999, AJ, 118, 2460

[7] Basri \& Reiners 2006, AJ, 132, 663

[8] Béjar et al. 1999, ApJ, 521, 671

[9] Bouvier et al. 2008, A\&A, 481, 661

[10] Burgasser et al. 2007, proceedings Protostars and Planets V, pp 427-441

[11] de Bruijne et al. 1997, proceedings ESA SP-402: Hipparcos Venice'97, pp 575-578

[12] de Wit et al. 2006, A\&A, 448, 189

[13] Caballero et al. 2007, A\&A, 470, 903

[14] Casali et al. 2007, A\&A, 467, 777

[15] Chabrier et al. 2000, ApJL, 542, L119

[16] Chabrier 2003, PASP, 115, 763

[17] Chappelle et al. 2005, MNRAS, 361, 1323

[18] Cutri et al. 2003, 2MASS All Sky Catalog of point sources

[19] Deacon \& Hambly 2004, A\&A, 416, 125

[20] Dobbie et al. 2002a, MNRAS, 335, 687 
[21] Dobbie et al. 2002b, MNRAS, 335, L79

[22] Dobbie et al. 2002c, MNRAS, 329, 543

[23] Gehrels 1986, ApJ, 303, 336

[24] Gonzales-Garcia et al. 2006, A\&A, 460, 799

[25] Jameson \& Skillen 1989, MNRAS, 239, 247

[26] Jeffries et al. 2004, MNRAS, 351, 1401

[27] Hambly et al. 1993, A\&AS, 100, 607

[28] Hambly et al. 1995, MNRAS, 273, 505

[29] Hambly et al. 2001, MNRAS, 326, 1279

[30] Hambly et al. 2008, MNRAS, 384, 637

[31] Hewett et al. 2006, MNRAS, 367, 454

[32] Kraus \& Hillenbrand 2007, ApJ, 664, 1167

[33] Kroupa 2002, Science, 295, 82

[34] Lawrence et al. 2007, MNRAS, 379, 1599

[35] Lodieu et al. 2006a, A\&A, 450, 147

[36] Lodieu et al. 2006b, MNRAS, 373, 95

[37] Lodieu et al. 2007a, MNRAS, 374, 372

[38] Lodieu et al. 2007b, MNRAS, 380, 712

[39] Lodieu et al. 2008, MNRAS, 383, 1385

[40] Lodieu et al. 2009, A\&A, 505, 1115

[41] Law \& Lunden-Bell 1976, MNRAS, 176, 367

[42] Martín et al. 2004, AJ, 127, 449

[43] Maxted \& Jeffries 2005, MNRAS, 362, L45

[44] Miller \& Scalo 1979, ApJS, 41, 513

[45] Moraux et al. 2001, A\&A, 367, 211

[46] Moraux et al. 2003, A\&A, 400, 891

[47] Pinfield et al. 1997, MNRAS, 287, 180

[48] Pinfield et al. 2003, MNRAS, 342, 1241

[49] Prosser 1992, AJ, 103, 488

[50] Preibisch et al. 1998, A\&A, 333, 619

[51] Preibisch et al. 2001, AJ, 121, 1040

[52] Preibisch \& Zinnecker 2002, AJ, 124, 404

[53] Reid 1993, MNRAS, 265, 785

[54] Reid \& Hawley 1999, AJ, 117, 343

[55] Salpeter 1955, ApJ, 121, 161

[56] Scalo 1986, Fundamentals of Cosmic Physics, 11, 1

[57] Skrutskie et al. 2006, AJ, 131, 1163

[58] Slesnick et al. 2006, AJ, 131, 3016

[59] Slesnick et al. 2008, ApJ, 688, 377

[60] Southworth et al. 2005, A\&A, 429, 625

[61] Stauffer et al. 1998, ApJL, 499, 219

[62] Tej et al. 2002, ApJ, 578, 523

[63] Tokunaga et al. 2002, PASP, 114, 180

[64] Walter et al. 1994, AJ, 107, 692 